\title{
Texto y contexto. Problemas y perspectivas en la filosofía intercultural ${ }^{1}$
}

\section{Text and context. Problems and perspectives in intercultural philosophy}

Recepción: Diciembre - 2008

Dr. Ricardo Salas Astraín ${ }^{\star}$

Aprobación: Mayo - 2009

\section{RESUMEN}

En este artículo destacamos un aspecto central del dinamismo de la racionalidad que brota al interior de la filosofía intercultural, en lo que refiere a la relación entre texto y contexto. Para ello tomaremos la discusión tal como se la encuentra hoy en las elaboraciones pragmático-hermenéuticas de filósofos tales como J. M. Ferry y M. Maesschalck, o interculturales como R. Panikkar y R. Fornet-Betancourt. La tesis principal es que al destacar los modos discursivos ellos aparecerán siempre vinculados a procesos culturales mayores y menores, donde todas las sociedades y comunidades humanas van afirmando, en su singularidad, unas formas renovadas y más elaboradas de su propia experiencia histórico-cultural. La relación de textos y contextos es capital porque a través de ella se puede dar cuenta cómo las culturas se abren a otras intermediaciones a partir de su mundo de vida.

PALABRA CLAVE: filosofía intercultural, texto-contexto, discursividad. intercultural philosophy, particularly as regards the relationship between text and context. To that effect we will take a look at current debates in the works of pragmatic-hermeneutic philosophers such as J. M. Ferry and M. Maesschalck, and in the intercultural arguments set forth by $R$. Panikkar and $R$. Fornet-Betancourt. The main thesis is that by highlighting discursive modalities they will always appear as linked to major or minor cultural processes. Here, all human societies and communities lay claim, in their singularity, to renewed and more elaborate forms of their own historical and cultural experiences. The relation between text and context is critical because through it reveals how cultures are open to futher intermediation from their life-world.

KEY WORDS: intercultural philosophy, text and context, discourse.

Decano Facultad de Ciencias Sociales y Jurídicas, Universidad Católica de Temuco Correo: rsalas@uctemuco.cl

1 Esta artículo recoge las conclusiones más importantes de mis dos últimos Proyectos de Investigación financiados por el Fondo de Ciencias y Tecnología de Chile, denominado "Discurso, contexto cultural y acción humana" (2001-2004 Fondecyt № 1010718), y el segundo, “Fundamentación teórico-práctica de la estructura de la experiencia humana” (2005-2007, Fondecyt 1050181). 


\section{¿Existe un foco filosófico intercultural?}

La 'filosofía intercultural' es un programa de trabajo actualmente vigente en muchos lugares del mundo, donde filósofos, pensadores y sabios se preguntan por el sentido del conocimiento en una era tecnocientífica, se preguntan por los alcances de los saberes humanos y cuestionan la primacía de la racionalidad filosófica y de la ciencia occidental, que han terminado sobreponiéndose a las diversas racionalidades y saberes presentes en la totalidad de las culturas y discrimina los saberes locales ${ }^{2}$. Refiere tal filosofía entonces no a una racionalidad falsamente universalista, sino a una racionalidad ampliada que asume sus limitaciones en el encuentro de "las razones de los otros", y que colabora en la recuperación de otras modalidades contextualizadas del saber y de la racionalidad que han sido excluidas y marginalizadas por una larga historia del racionalismo cartesiano. Una de las principales conclusiones a la que llega una filosofía de este tipo es que la razón no existe en forma unitaria ni homogénea en ningún universo cultural ni civilizacional determinado, sino que ella siempre se va modulando polifónicamente, abierta a variados modos de articulación de conocimientos y saberes culturales que brotan todos ellos de vías para responder a las experiencias humanas. Por ello hasta el minuto en este programa se han elaborado categorías tales como interlogos o polilogos ${ }^{3}$.

Esta concepción plural de la razón es capital porque no solo es un presupuesto de la filosofía intercultural, sino que éste se transforma en el nuevo punto de partida de la reflexión de la pluralidad cultural: no solo como encuentro de culturas, sino como encuentro de saberes y de prácticas. Los modos discursi- vos culturales nos servirán de apoyo para plantear, con mayor firmeza filosófica, la cuestión del vínculo teórico-práctico entre los textos y los contextos, que también están definidos por la heterogeneidad ${ }^{4}$.

Para la filosofía intercultural, el análisis de los textos redefine de otro modo la conciencia de la diversidad cultural, y se plantea la interrogante siguiente: ¿cómo a través de este nexo problemático se puede intentar reconstruir unos conceptos que sean suficientemente operatorios para avanzar en campos específicos de la literatura, de la educación, de la política etc.? No basta solo con evidenciar unos presupuestos generales acerca de los textos y contextos en el marco de la racionalidad filosófica intercultural, sino que también es menester dar cuenta de otros cruces teóricos, basados principalmente en el ejercicio de las ciencias socioculturales críticas y sobre todo de la recuperación de la experiencia social. Dicho de otro modo, las categorías que necesitamos hoy para desenvolver este lazo entre texto y contexto debieran no ser tan abstractas, sino que ellas debieran permitirnos, en principio, ensamblar y reconstruir las diversas formas del discurso inherentes a las culturas desde sus raigambres histórico narrativas hasta sus formas de reconstrucción comunicativa más complejos a partir de una concepción de la dinamicidad de la significatividad humana abierta a la interacción cultural. Este me parece que es el trasfondo de la cuestión intercultural del vínculo del texto y del contexto que debiéramos explicitar aquí.

Desde tales presupuestos teóricos es posible converger con la filosofía occidental contemporánea dado que la problemática del texto y del contexto cabe situarla claramente

2 Para tener una idea de la amplitud y riqueza de este planteamiento, se puede consultar los diferentes trabajos compendiados en el sitio Internet: www.polylog.org Se puede ver la síntesis que hago de algunos de estos problemas en un artículo: http: / / them.polylog.org/5/asr-es.htm

3 En nuestra concepción de la filosofía intercultural hemos hecho referencia a nociones tales como interlógico o polilógico, las que mantienen aún una enorme deuda con la matriz filosófica greco-latina que dio históricamente origen en Occidente a la ligazón entre razón y lenguaje. Sostenemos que en trabajos filosóficos futuros cabría asumir al menos otros análisis para elaborar categorías acordes a las principales matrices y/o tradiciones de los grandes troncos lingüísticos de la humanidad, más allá de las posibilidades lingüísticas del antiguo griego y del latín.

4 Se sabe que este tema filosófico es una problemática que ha ocupado en obras conocidas a distintos filósofos del siglo XX, pensadores alemanes y franceses como Gadamer, Habermas, Ricoeur Derrida, Foucault, los rusos Propp y Bajtin, y en América Latina Schwartzmann, Kusch y Roig. Todos ellos han legado distinciones teóricas importantes que entregan algunos soportes que no desarrollaremos específicamente aquí. 
al interior del denominado "giro lingüístico" y que converge con los desarrollos diversos aportados por las ciencias del lenguaje. Todas estas convergencias son fruto de una lenta elaboración que ha sido uno de los aportes más significativos de las ciencias humanas occidentales en este último siglo. Al asumir que razón y lenguaje requieren esclarecerse mutuamente, parece implicar una primacía del paradigma del lenguaje humano, lo que la tradición helena denominó Logos. Pero lo que se requiere es considerar la riqueza, la complejidad y la diversidad de todas las denominaciones que refieren al modo de tensionar el significar en una relación de apertura y de cierre. En otras palabras, se trata en nuestro enfoque de no proseguir de ningún modo un ideal logocéntrico, en su sentido reductivamente helenístico, y decir que el lenguaje en su sentido más amplio y variado es el único que permite repensar la dinámica de las racionalidades al interior de las culturas, ya que es el intermedio en el que se puede replantear dicha relación con las otras culturas y sus contextos. Afirmamos que las culturas se definen por lenguajes plurales y diversos, que nos entregan las claves necesarias para descifrar lo que son los juegos entre los textos y sus contextos.

En este sentido, seguiremos utilizando el término que se ha hecho conocido en este legado griego como logos (lenguaje, razón). Esta noción del logos, la abriremos gradualmente a su más amplio campo semántico, reconociendo que éste está limitado geoculturalmente y que implica muchas dificultades para comprender otras formas discursivas ${ }^{5}$. Para nosotros, será aceptado solo en tanto esos núcleos significativos que aparecen en nuestra tradición de la filosofía y la ciencia occidentales en que nos hemos formado, y solo los valoramos porque nos aportan unos índices que permiten iniciar el camino de reconstrucción de los textos y de los contextos ${ }^{6}$. Sería clave redefinir el asunto del mythos y el logos como ya lo hemos indicado en otros trabajos citados, la discursividad en clave intercultural, y el complejo problema de la traducción porque en estos temas se anudan los principales desafíos de la teoría del discurso, y donde se resuelven en un cierto sentido el proyecto discursivo de la razón y la dinamicidad del lenguaje ${ }^{7}$.

Empero, esto no es solo un asunto filosófico general como lo han demostrado todas las ciencias del lenguaje. Se puede encontrar en la historia de la humanidad una prueba de la diversidad lingüística, ya que no existe una lengua común de la humanidad. Por otra parte, todos los lenguajes de la humanidad conocidos, se han ido constituyendo en un rico proceso de gestación de formas siempre plurales posibilitadas por la estructuración del lenguaje mismo, y esto acontece tanto hacia el interior de la propia comunidad lingüística como en su interacción con otros lenguajes.

\section{El lenguaje plural: los textos y los contextos en tensión}

Es algo obvio sostener que las sociedades humanas desde sus orígenes remotos han hablado lenguas diferentes, ya que no existen antecedentes de que la humanidad haya vivido en un momento paradisíaco de una única lengua universal. Asimismo, se entiende que en esta historia de complejos vínculos que unen a las miles de lenguas históricas entre sí, han existido procesos histórico-culturales, políticos que han difundido las lenguas más allá de los límites de la comunidad hablante; ello ha condicionado la relación de las lenguas en los muchos procesos de dominación, de eliminación y de mezclas lingüísticas que ha vivido la humanidad. Por ello, todos los esfuerzos de comprensión de cierre y apertura de las comunidades lingüísticas no pueden prescindir del análisis de esta relación socio-cultural y polí-

5 Tomamos el concepto lugar filosófico como espacio condicionante a partir del sentido que aparece en la obra Esbozo de una antropología filosófica, en Obras Completas de Rodolfo Kusch, Tomo III, pp. 381ss. Agradezco esta indicación de Cristian Valdés que elabora una tesis doctoral en el Centro de Filosofía del Derecho de la UC de Lovaina.

6 Es posible en este terreno hacer un diálogo con la obra de W. Mignolo, es especial en relación a la imbricación entre lenguas, literaturas y conocimientos. Cf. pp. 291ss. Agradezco a Pablo Juan Bermúdez estas referencias a Mignolo.

7 Cf. Ricardo Salas, "Problemas y perspectivas de la filosofía como diálogo intercultural” en Revista Brocar N²7 (2003), pp. 275-294 
tica entre las lenguas. Nos encontramos casi siempre frente a una cierta paradoja acerca de la tendencia a la totalización de las lenguas y la fragmentación que les afecta a ellas desde su mismo interior. Todos los esfuerzos del romanticismo histórico por reducir las lenguas a un solo árbol que las ordene se han mostrado infructuosos, y asimismo todos los intentos por demostrar la inconmensurabilidad de las lenguas se han enfrentado al fracaso, porque aunque existen lenguas diferentes, gracia y a pesar de ellas, los seres humanos han logrado casi siempre comunicarse en esta larga historia de tensiones y conflictos. Todo ocurre como si el lenguaje llevara en sí mismo los dispositivos de la diferenciación y de la articulación sin poder reducirse a uno $u$ a otro. Estas consideraciones cabe hacerlas en relación a los textos. ¿Qué vamos a entender por texto aquí? ${ }^{8}$

Hagamos algunas distinciones más precisas. Una primera aclaración: el texto no se debe identificar con el ejercicio de la escritura en su sentido reducido, el texto no es un corpus de signos escritos en cualquiera soporte, es decir, no alude exclusivamente a un producto de vocabulario determinado por los procesos de escritura codificada por las grandes lenguas de la Humanidad (un jeroglífico egipcio o maya, un código en tabletas o en formas diversas de escritura estabilizadas de signos escritos: pergaminos, rollos o libros como los conocemos). En nuestra perspectiva, un texto es una textura o entramados de significados y sentidos que lo podemos reconocer en sus formas orales, escritas y/o icónicas, por señalar tres formas reconocidas en la cultura occidental.

Las culturas humanas, como se sabe, son eminentemente orales e incluso a pesar del enorme esfuerzo de la escolarización y de la preeminencia de lo escrito, se podría decir que lo esencial de la experiencia humana en las culturas humanas, variadas y heterogéneas, no se transcribe ni se codifica. Sabemos que son solo algunas pocas que en el curso de su evolución han llegado a la fase de escribir sus mitos y narraciones, sus legislaciones, y sus contra- tos, lo que en muchos casos tiene que ver con la dinámica de las estructuras socio políticas que las gobernaron y gobiernan. Incluso, es menester reconocer que hasta los tipos de lenguaje practicados masivamente en muchas de nuestras culturas modernas, como en las que nosotros habitamos, comportan grandes dosis de oralidad y narratividad que siguen siendo el soporte de nuestras interacciones. Bastaría indicar que la mayor parte de nuestros diálogos significativos y de nuestras discusiones culturales no se escriben. Un ejemplo de esto acontece en las sociedades contemporáneas en el enorme despliegue de los procesos icónicos derivados de los actuales sistemas mediáticos que los originan (publicidad, graffitis, filmes, etc). La escritura entendida como texto escrito en sentido reducido se exige siempre de cara a la institucionalización legal de la vida social, tal como acontece en: un contrato de compraventa, un permiso de trabajo, lo que asume la forma de una prueba escolar o de una validación exterior a la interacción de los sujetos, etc., pero no se encuentra ahí el principio mismo de la significatividad cultural. El texto transcrito siempre es posterior en relación a las exigencias de la oralidad.

Una segunda aclaración: el texto, según como se concibe en la filosofía intercultural, tal como la entiendo, es una suerte de paradigma de obras culturales que refieren a tramas y a estructuras de signos en un sentido amplio que son significativos para los sujetos portadores de una cultura específica. Por ello el texto será entendido aquí, en general, como aquel dispositivo de una cultura específica que articula significados y sentidos que pueden ser explicados y comprendidos por los usuarios del mismo lenguaje y eventualmente por otros que pueden y quieren aprender a descifrarlos y a interpretarlos. Esto no quiere decir de ninguna manera que todos los sentidos y significados pueden ser comprendidos siempre a cabalidad. En este sentido, el texto es un dispositivo en principio abierto y no cerrado a la interacción, lo cual es válido para las culturas orales y/o escritas, y para las que se ubican en unas y otras, como asimismo a las formas icónicas en las culturas mediáticas emergentes.

8 Cf. P. Ricoeur, ¿Qué es un texto?, en Del Texto a la Acción, 
En este sentido, aunque sea redundante decirlo: todos los textos son siempre culturales, derivan de formas de vida específicas que aluden a determinados saberes y prácticas de la humanidad; no existen en una primera instancia textos de suyo universales, lo que existen son textos orales definidos por contextos específicos, que pueden entrar en un procesos de universalización por una transcripción posterior, y que pueden llegar a ser portadores de identidades transversales para muchas culturas, como ocurre frecuentemente con los textos sagrados de las religiones de libro, donde nos referimos a lenguas que ya no se hablan, y que requieren de especialistas en filología y en lenguas cuyos sentidos no son descifrables para la mayoría. Esto es lo que hace teóricamente interesante a los textos de los proyectos religiosos universales.

En conclusión: un texto admite un ad intra que afirma una dinámica identitaria cultural de origen -frecuentemente de raigambre oral- y un ad extra, que refiere a modos de reconocimiento alterativo por miembros de otras culturas que no comparten el mismo origen. Pero entender esta singular interacción cabe admitir que un texto necesite de un contexto, el contexto es un conjunto de aspectos que necesitamos considerar para dar cuenta de la especificidad del texto, pero ello no es nunca simple, ya que existen tipos de contextos, en lo que refiere a los contextos más conocidos: psicológicos, sociales, políticos, culturales, etc. que están siempre en juego en la cultura originaria de los textos como en la cultura que se reconoce en ellos. Decir esto implica que si se pretende un análisis de un texto es preciso aludir a un contexto, eso permite elucidar al interior de un texto varios contextos culturales diferentes (que van de lo particular a lo universal), de este modo poder entender un texto al interior y al exterior de una misma cultura, exigiría mostrar las relaciones con la ampliación de los contextos más particulares hasta sus niveles más universales.

Esto lo puede ejemplificar lo que llamamos un mito. Lo que él significa entonces no se define por una sola versión, no es posible admitir que un texto mítico contenga un solo contexto en la cultura de origen, por ello todos los denominados mythos presuponen muchas versiones, el texto de un mito no existe jamás separado de sus versiones, lo que quiere decir que al interior de una misma lectura hay diversas posibilidad de "leer" una obra para captar sentidos y significados específicos, que son desconocidos para los iniciados en esa cultura. Ello refiere de algún modo a la idea de tradición, que es utilizada fuertemente en hermenéutica. Y si consideramos esta doble contextualidad sabemos que los mitos son siempre recreados por situaciones particulares ligados a sujetos y comunidades específicas, que en verdad refieren a una estructura social cada vez mayor.

Al mismo tiempo, el ejercicio de una comprensión ad extra permite revisar otro ejercicio de las variadas lecturas posibles. Desde otros horizontes histórico-culturales que implican contextos disímiles que no son necesariamente los mismos que establecieron los textos en el espacio y en el tiempo propio, por ejemplo, como se han "leído" los textos africanos, hindúes y chinos en una tradición colonial o neocolonial. Es preciso indicar que una cultura occidental dominante ha pretendido casi siempre definir una lengua canónica y/o textos canónicos ya que ha buscado disminuir las plurales tradiciones e interpretaciones rivales presentes en los textos. Lo que separa un análisis intercultural actual de los intereses anteriores de los insignes estudiosos de otras culturas "extrañas" y "exóticas", es que en la actualidad nos interesa mucho más ese juego de sentidos y significados que se obliteraron, invisibilizaron y escondieron, para mostrar las otras posibilidades que contenían lenguas y textos. Pero también reconocer que en el ejercicio alterativo de la mayor parte de nuestras lenguas y textos occidentales son extraños y exóticos para otros sujetos de culturas diferentes.

Por ello, los textos y contextos tienen una relación que pasa por la interacción de los mundos de vida, a saber, si buscamos comprender un texto, en un paradigma reducido de la escritura, ello nos exige comprender tanto el lugar del autor (es) que está en el origen de la trama de sentidos y asimismo una recontextualización de parte del lector(es) local(es) y extranjero(s). Los textos cobran y recobran sentido siempre en lo que se dice en una expresión germana "Sitz im Leben", desde donde ellos plasman su configuración interna 
y externa, que abre mediaciones significativas y desde donde podremos eventualmente reconocerlos. En otras palabras, la articulación entre textos y contextos es crucial porque se juega en ella la posibilidad de la intercomunicación.

Empero ello presupone, desde mi perspectiva filosófica intercultural, no solo una teoría de los textos que asuman las diferencias culturales, de una teoría de la Inter.traducción, de una teoría intercultural de la lectura, en definitiva de una teoría general del lenguaje humano abierto a la diversidad cultural (de la cual disponemos hoy), sino en algunos de sus trazos más relevantes, pero no de un modo completo.

Pero al plantearse la cuestión epistemológica teórica y/o disciplinaria, ¿qué enfoque o perspectiva define de mejor modo estas multidimensionalidades textuales y contextuales? Desde mi perspectiva filosófica me parece que, aunque contamos con avances importantes en las disciplinas generadas por Occidente, tales como la etno-historia, la antropología, la semiótica y la pragmática, entre muchas otras, el problema intercultural difícilmente ha podido resolverse: no solo la historia, sino el análisis literario, la semiótica, la semántica y la pragmática han querido definir el análisis de los textos y de sus contextos desde sus propias prerrogativas racionales, donde sus configuraciones explicativas, con mucha frecuencia, aparecen asociadas a los modos cientificistas occidentales de hacer ciencias. Para el campo que nos interesa: dentro de los historiadores occidentales no es aún asumido plenamente un enfoque de reconstrucción de la historia por sujetos y comunidades que no sean los historiadores profesionales; pesa siempre el problema de la crítica, de la validez, etc. En el campo del análisis literario, y las demás disciplinas del lenguaje, acontece algo parecido. Los especialistas avanzan en sus explicaciones de los procesos de los autores y de las comunidades parlantes. Pero yo creo que el asunto escapa en verdad a cada una de las disciplinas específicas, porque el análisis de las tramas y estructuras discursivas en lenguas orales y escritas (e icónicas) es clara y definitivamente interdisciplinaria, que es justamente una de las faltas mayores de la episteme occidental. Por ello, apoyado en el giro lingüístico, diría de un modo abstracto, que el texto y el contexto refieren siempre a cruces interdisciplinarios y a una tensión filosófica entre hermenéutica y pragmática.

En otras palabras, el texto y el contexto están casi siempre atravesados por un problema epistemológico que la hermenéutica crítica europea ha denominado "la lucha de las interpretaciones". Este rico campo de disputa abre la discusión de la multidimensionalidad semántica de todos los textos culturales a su interacción con los otros textos, me parece que es otra clave para comprender un análisis intratextual e intercontextual como el que se plantea en el contacto entre culturas.

Cuando me refiero a la "intratextualidad" quiero indicar que el texto presupone siempre una serie de distinciones al interior de las tramas y estructuras significativas en que fue formulado, e "intertextualidad" refiere por su parte a que todos los textos siempre van más allá de su propia cultura, sociedad e historia de origen. Están disponibles para otros que sepan leerlos y comprenderles y asumirles en su propuesta de sentido y significado, por ejemplo: libros sagrado escritos como la Biblia, el Corán y el Bhagavad Gita están todos ellos conformados por muchas tramas internas generadas por "formas discursivas" creadas en las lenguas en que fueron pensados, pero a la vez ellos pueden ser leídos, releídos, traducidos y entendidos desde contextos muy diversos.

Pero el asunto teórico de una interculturalidad textual es mucho más compleja todavía, porque las categorías de texto y de contexto, de intratexto y de intercontexto, aun siendo específicas, apuntan a un campo de la diversidad cultural donde todas ellas se relacionan entre sí; todas aluden a una experiencia humana plural, que recién la reflexión intercultural comienza a asumir seriamente, y por tanto configuran categorías que no solo son aplicables a los grandes textos escritos de las grandes civilizaciones como lo demuestran las exégesis y hermenéuticas bíblicas y coránicas, sino también a todas las narraciones que encontramos en el enorme abanico de culturas existentes. En este sentido no bastan las narraciones canónicas definidas por Occidente, sino que se requiere considerar todas las narracio- 
nes denominadas "míticas" y "narrativas" en que las culturas ofrecen dispositivos para que sus miembros cuenten sus experiencias.

Ello demuestra que las categorías del texto y del contexto no refieren solo a los cánones precisados por una razón científica, sino que contienen una dimensión teórica práctica. La experiencia y la acción humanas, al ser analogadas con el paradigma lingüístico, permiten remontar la estructuración del lenguaje mismo, desde una estructura objetiva la lengua hacia una estructuración subjetiva e intersubjetiva el discurso, por lo que es posible descubrir la génesis de las significaciones tanto en el proceso de elaboración de los lenguajes de las ciencias como en los diversos niveles de significación de las formas discursivas culturales que buscan decir la experiencia y la acción humana en sus contextos y en su búsqueda de universalidad.

De modo que, así como la forma lingüística habita en la dinamicidad del lenguaje nunca clausurado, la acción humana puede analogarse también al juego entre un texto y un contexto, texto como expresión del acto de una existencia cultural, ya que puede asegurar su propia identidad a partir de una reapropiación reconstructiva de sí misma; y contexto porque permite el proceso de reelaboración desde lecturas reiteradas asegurar la reconstrucción del ejercicio del reconocimiento. Esto es clave porque a través del nexo texto-contexto nos encontramos con el paso que se necesita para pasar desde lo histórico a lo universal, ya que no existe la experiencia humana en abstracto, y tampoco lo humano en general, sino siempre las experiencias de los seres humanos y los vínculos interhumanos que ellos pueden generar. En todo ello, se juega la capacidad de los textos y de los contextos para inducir una creciente reflexividad de los sujetos.

\section{El problema intercultural de la reflexividad}

El cruce entre una hermenéutica y una pragmática del discurso que destacamos al inicio permite considerar, entonces, desde una perspectiva más adecuada el vínculo entre texto, contexto cultural y acción humana; esto exige explicitar una perspectiva que nos llevó a elaborar una teoría discursiva de los textos y de los contextos culturales conflictivos. La elaboración de esta propuesta es lo que ya denominamos hace pocos años en la "Ética Intercultural", como:

"aquella forma de tematización discursiva del ethos al interior de una cultura, a partir del despliegue de los diversos niveles de reflexividad que se alcanzan en sus formas discursivas, y los posibles espacios de apertura comunicativa que se abren frente a otras formas de reflexividad asociados a otros contextos culturales"

Esta definición es claramente deudora de un enfoque que considera el lenguaje en que se vierte la experiencia y los diversos sistemas de significación y sentido que tienen los enunciados en una cultura específica y que pueden ser muy variados: preceptos, consejos, relatos, reglas, prohibiciones, etc. Esto tiene que ver con el concepto hermenéutico y pragmático actual, que considera que los valores y normas son estructurados a partir de registros del lenguaje, donde encontramos reglas lógicas, semánticas y pragmáticas, que aseguran a sus usuarios las necesarias interrelaciones simbólicas y pragmáticas básicas. Esto supone que al interior de los discursos de una cultura siempre encontramos un vínculo entre lo específicamente propio y las exigencias universales, encontraremos, por lo tanto, en su ethos referencias a lo histórico y lo universal como logos.

Estas ideas presuponen que existen cuatro problemas principales del vínculo texto, contexto y acción.

1.- El conflicto de las interpretaciones de los textos culturales aparece fundamental en la comprensión y deliberación humana y por tanto el análisis contextualizado de los textos de una cultura es clave para la comprensión de la acción humana en sociedades específicas, frecuentemente asimétricas, porque plantea

9 R. Salas, Ética Intercultural, p. 84. 
por ejemplo preguntas tales como: ¿cuáles son los textos mayores y los textos menores?, ¿es posible afirmar un canon único?

2.- El diálogo intercultural entre los diversos textos en interacción debe entenderse siempre en espacios plurales y siempre frente a contextos heterogéneos y asumiendo formas dispares y plasmándolos en niveles discursivos diversos, lo que exige siempre el proceso de una compleja recontextualización histórica de todos los textos: ¿por qué en un país como Chile sus textos están definidos desde la metrópoli y la sociedad mayor?, ¿cuántos textos que se ofrecen a los niños y jóvenes estudiantes están completamente descontextualizados?

3. - La reflexividad propia de los textos que refieren a la acción humana en un marco intercultural, deberían ser relacionados con los registros discursivos inherentes a las culturas específicas, si se quiere comprender y enfrentar tanto los conflictos internos de la propia cultura en transición como los eventuales conflictos externos frente a las culturas en contacto; ella posibilita la convivencia con los otros.

4.- La interculturalidad textual, como búsqueda de este espacio de comprensión "entre-culturas", supone una opción por generar nuevas formas de una interdiscursividad que valide un procedimiento definitivo de las reglas, a partir de una modalidad contextual que valida el diálogo y haga inaceptable el recurso del fundamentalismo a la violencia y a la guerra.

\section{Cuestiones fenomenológicas finales acerca de los textos y contextos}

Antes de terminar hagamos algunas breves consideraciones fenomenológicas de los textos. En esta perspectiva dinámica el análisis del texto y del contexto requiere de otra categoría central que nace del pensamiento tardío de Husserl, la del "mundo de la vida" (Lebenswelt). Desde este entorno vital, social-cultural e histórico específico brotan todos los sentidos y significados de las experiencias originarias sobre las que se establecen los dispositivos de sentido de los textos. La acción humana está, por tanto, al mismo tiempo inserta en un mundo simbólico humano que solo accede parcialmente a la apropiación de este esfuerzo por existir, y por consiguiente, debe recurrir a la universalización comunicativa de sus contenidos culturales.

Esta categoría del mundo de la vida, levantada por el último Husserl en su crítica a los tipos de epistemologías reduccionistas del siglo XIX, hoy es retomada por los filósofos de la hermenéutica y pragmática, ya que es el ariete de la crítica a la racionalidad objetivadora y dominante iniciada por el positivismo, el naturalismo, el psicologismo y el historicismo predominantes en el siglo XIX, y que se extiende ciertamente al análisis cientificista del lenguaje. La cuestión filosófica más abstracta del diálogo intercultural e intertextual, que hemos planteado aquí, es descubrir cómo esta noción del mundo de la vida permite una revisión profunda del lenguaje y de las lenguas de la humanidad, y permite avanzar en una crítica sistemática a la lectura científica moderna de los textos, pero mucho más allá de las limitaciones provincianas de dichas teorías.

Asimismo, el reconocimiento del mundo de la vida de los otros diferentes, que no trasunta solo una cuestión epistémico acerca de los textos, sino que alude al sentido eminentemente ético y político que ellos tienen, porque lleva a revalorizar el sentido del mundo cultural de los otros, y abre la posibilidad de la comprensión de otros mundos culturales. Es relevante mostrar cómo una noción fuerte de diálogo intercultural, inspirada en estas categorías, es cuestionadora de los lenguajes abstractos propios de los procesos tecnocientíficos, que definen un tipo de racionalidad homogeneizadora por sobre el despliegue de las narrativas. Los actuales problemas y perspectivas de la filosofía como diálogo intercultural refieren a una crítica de este modelo monocultural de los textos que ha predominado en Occidente, y a la consagración de un canon cultural de sus textos-emblemáticos, pero soslaya y desconoce el valor de los otros textos culturales y sobre todo desvaloriza sus respectivos contextos.

Esta tesis central permite visualizar, de modo claro, el relieve que asume el vínculo entre la hermenéutica y la pragmática del discurso ya que ambas permiten dar cuenta de la mutua acción intercultural entre sujetos y 
textos culturales que exige repensar el programa de la filosofía intercultural. Ello permite asumir un papel relevante en la comprensión de los otros textos "extraños" y "extranjeros", asegurando las condiciones mínimas y procedimientos básicos sobre los que se levanta un verdadero diálogo intercultural de la comprensión de los otros textos y contextos. La filosofía intercultural asume este complejo desafío, asegurando la posibilidad de una verdadera intercomprensión mutua, no a partir del conjunto de códigos discursivos más poderosos, sino del esfuerzo de un interlogos que sugiere el ensamblaje de vías que permiten la eventual intersección de dos o más conjuntos de códigos discursivos. Esto es lo que cabría denominar el auténtico diálogo intercultural que es parte de la vocación actual de la filosofía ${ }^{10}$.

La estructura general de este esquema a partir de la filosofía intercultural, a diferencia de la visión de la razón de algunas teorías modernas universalistas o posmodernas hoy tan de moda en el énfasis de las diferencias, propone un concepto de interculturalidad textual, que implica exponer una tensión interior a la dinámica de los encuentros y desencuentros de los textos y de los contextos a partir de encuentros interdiscursivos al interior $y$ al exterior de las culturas, por ello el asunto no es nunca oponer la universalidad versus la contextualidad.

El privilegio del discurso que hemos realizado en este modo de entender la filosofía intercultural no supone quedarse en el mero terreno de textos definidos lingüísticamente, sino que ellos nos devuelven a los mundos de vida, donde aparece el lenguaje y la acción humana en toda su riqueza sinérgica y multifacética. Texto y contexto no pueden ser disociados, pues el discurso de la vida precisa de la formalidad del lenguaje y de su sustantividad ético-cultural. Esto hace inviable separar el logos del ethos de las formas expresivas concretas en que se expresan las culturas concretas.

1.- Las tradiciones textuales pluriétnicas, en términos generales, aseguran el orden ideológico de cada cultura, por ello las formas del imaginario regional están impregnadas de símbolos, textos e imaginarios que señalan lo propio y lo ajeno de esta compleja historia fronteriza. Ellos nos entregan distinciones para pesquisar el origen ideológico de los conflictos interétnicos en todos sus registros: sociopolíticos, socioculturales, etc.

2.- Todos los relatos míticos y los discursos históricos muestran una pluralidad de las formas concretas: ninguna sociedad, cultura ni religión es un todo homogéneo y compacto, ella supone el juego de formas discursivas y tradiciones plurales. La recontextualización de los textos indígenas, por ejemplo, supone encuentros de convergencia-divergencia.

3.- Las ciencias humanas como ejercicios reflexivos son parte de una dinámica que asegura la posibilidad de la reconstrucción de los sentidos culturales, por ello, en esta etapa se puede asegurar con más claridad el diálogo interlógico, que supone un ejercicio complejo de inter-traducción entre los conocimientos y los saberes de las culturas en diálogo.

4. - La filosofía intercultural en la medida que ejercite la interdiscursividad de sus tradiciones ad intra, puede colaborar a un diálogo efectivo con otros discursos, ya que el cierre sobre sí mismo es etnocéntrico, y puede conducir al fundamentalismo, que vincula poder político y poder cultural en un todo. Esto no ayuda al momento ad extra que exige una visión intercultural abierta a los otros textos. 


\section{Bibliografía}

ARPINI, A. (2000) Razón práctica y discurso social latinoamericano, Mendoza, Ediunc.

FORNET BETANCOURT, R. (2003) Interculturalidad y Filosofía en América Latina, Concordia Band 36, Aachen.

FORNET-BETANCOURT, R. (2006) La interculturalidad a prueba, Concordia Band 43, Aachen.

JACQUES F., (2006), De la Textualidad. Para una textología general y comparada, Santiago de Chile, Ediciones PUC de Santiago.

KUSCH, R. (1990) Esbozo de una antropología filosófica americana en Obras Completas, Editorial Fundación Ross, Rosario, Tomo III.

MIGNOLO, W. (2003) Historias locales/diseños globales. Colonialidad, conocimientos subalternos y pensamiento fronterizo. Madrid, Ediciones AKA.

MALIANDI, R. (1984) Cultura y Conflicto. Buenos Aires, Editorial Biblos.
PÉREZ ZAVALA, C. (1997) La filosofía latinoamericana como compromiso, UNRC-Ediciones del ICALA, Río Cuarto.

RICOEUR, P. (2001) Del texto a la acción. MéxiCO, FCE.

ROIG, A. (1981) Teoría y la crítica del pensamiento latinoamericano. México, FCE.

SALAS, R. (2003) Ética Intercultural. Santiago de Chile, Ediciones UCSH.

SALAS, R. (2006), "Ética, Políticas del Reconocimiento y Culturas Indígenas" en Estudios Interculturales, Hermenéutica y Sujetos Históricos, Santiago de Chile, Ediciones UCSH.

SAUERWALD, G. (2008) Reconocimiento y Liberación. Axel Honnett y el pensamiento latinoamericano, Berlin, Lit Verlag.

Varios Autores (2009). Interculturalidad crítica y descolonización, Instituto Internacional de Integración, La Paz. 